\title{
Correlative Computational Image Analysis of Blood Drop Drying Patterns
}

\author{
Sunanda Sharma ${ }^{1}$, Neri Oxman ${ }^{1}$ \\ 1. Media Lab, Massachusetts Institute of Technology, Cambridge, USA.
}

Blood, in its wet or dried form, has been central to standard laboratory tests for a myriad of conditions and diseases in humans and other animals for decades. In this time, the amount of blood required to conduct procedures has decreased, and the method of obtaining the sample has become more streamlined. However, a comparatively recent line of work suggests that multiple types of useful diagnostic data may be collected from a single drop through examination of crack patterns created through desiccation.

Blood is a colloid, and as such creates complex patterns upon drying [1]. These patterns have been generally characterized, with studies conducted on the physical forces that underlie specific crack types [2] as well as overall pattern of cracks and folds and the dynamics of fracture [3]. The field is of interest because it is known that the composition of colloids greatly affects the resulting crack pattern, and thus, a single drop could contain a vast amount of extractable information through simple microscopy. While previous papers have proposed the diagnostic potential of this method for specific disorders [4], the present study aims to illustrate that more subtle differences in composition can also be detected through more powerful image analysis. Furthermore, we identify several key observations that can be used to automate the image processing and analysis method, and serve as a foundation for application of computer vision and machine learning techniques.

In this study, cracking patterns as a function of blood glucose were modeled and evaluated. Finger pricks were used to collect $400 \mu \mathrm{L}$ of whole human blood, which was collected in a $1.5 \mathrm{~mL}$ tube and used immediately. Whole blood was mixed in a 1:1 solution with 1X PBS (Corning), held at physiological $\mathrm{pH}$ (7.4). The amount of blood glucose in all samples was measured using a commercial blood glucose meter (Ascensia). Experimental model solutions of blood were created using different quantities of filter-sterilized $100 \mathrm{mmol} / \mathrm{L}$ dextrose solution (VWR Analytical). Samples at glucose concentration $2.72 \mathrm{mmol} / \mathrm{L}, 5.99 \mathrm{mmol} / \mathrm{L}, 27.3 \mathrm{mmol} / \mathrm{L}$, and $30.58 \mathrm{mmol} / \mathrm{L}$ were created and categorized as "low-normal" (under $7.8 \mathrm{mmol} / \mathrm{L}$ ) and "high" (above 10mmol/L). Glass coverslips were cleaned with $70 \%$ ethanol and dried in a biosafety cabinet under ultraviolet light. $10 \mu \mathrm{L}$ of sample was pipetted onto the surface of each coverslip and immediately visualized using a WILD Makroskop M420 and captured with a Nikon D3300 camera. Each drop imaged after a fifteen-minute drying period. All experiments were conducted at room temperature $\left(21^{\circ} \mathrm{C}\right)$ and normal relative humidity $(\sim 20 \%)$.

A base set of whole blood drops were imaged in different quantities ( $3 \mu \mathrm{L}, 5 \mu \mathrm{L}$, and $10 \mu \mathrm{L})$ to select the optimal volume in terms of drying time and full pattern formation. Drops smaller than $10 \mathrm{uL}$ did not achieve three distinct regions: peripheral, coronal, and central, and thus could not be used for analysis. Images were first pre-processed to increase exposure, and then converted to binary format. From initial visual observation, one key feature of interest were radial cracks that formed perpendicular and immediately adjacent to the perimeter of the drop. The quantity, length, and width of these cracks varied between the "low-normal glucose" samples and the "high glucose" samples. Furthermore, the angles formed between cracks in the "high glucose" samples more often ranged between $80-100^{\circ}$. These preliminary observations were quantified using the Fiji toolkit (NIH), and indicated a positive 
correlation between glucose concentration and radial crack frequency, and an inverse correlation with radial crack width. Computational analysis was then continued using a Python-based version of OpenCV, which allowed for rapid and automated characterization of image features such as lines, coloration based on pixel value, and local patterning in each of the three drop regions. Detection and quantification of these features can be done through leveraging a combination of off-the-shelf image analysis techniques, such as binary thresholding, Canny edge detection, and the Hough transform, with custom algorithms.

This work sets a firm foundation for extending the possibilities of computational image analysis by using machine learning techniques, specifically, Convolutional Neural Networks. Future experimentation will focus on controlling experimental conditions, such as drop deposition and shape, as well as the impact of other measurable variables, such as $\mathrm{pH}$, oxygen content, and substrate characteristics. In addition, video input will be explored to garner data such as crack formation rate. This approach can likely be extended to detect many other common compounds typically tested in metabolic, hormonal, and electrolyte blood panels, as well as considered for use with other biofluids, such blood plasma or sweat. Correspondingly, a comprehensive suite of computational techniques can be created to analyze a range of tests conducted with inexpensive and accessible tools and materials, and enabled through light microscopy [5].

\section{References:}

[1] R. D. Deegan et al, Nature 389 (1997), p. 827.

[2] R. Chen et al, Journal of Materials Chemistry B 5 (2017), p. 8991.

[3] B. Sobac and D. Brutin, Colloids and Surfaces A 448 (2011), p. 34.

[4] R. Chen et al, Advances in Colloid and Interface Science 231 (2016), p. 1.

[5] Acknowledgements: This work was generously supported by the Media Lab Consortium, GETTYLAB, and the Robert Wood Johnson Foundation. The authors also acknowledge J. S. and S. S. for helpful discussions.

a

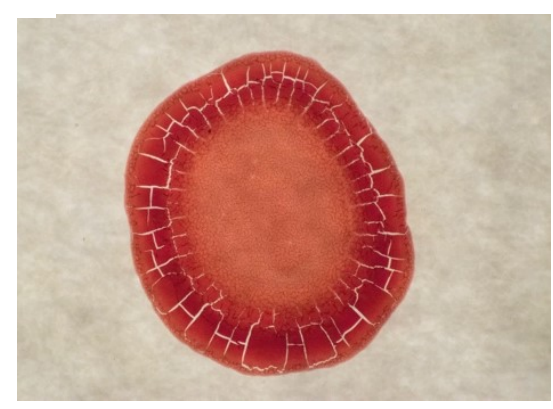

$\mathrm{b}$

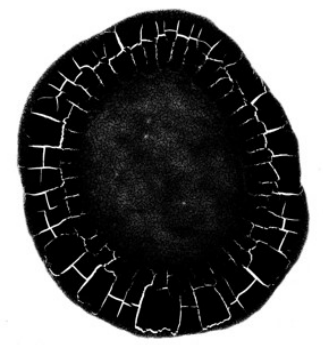

$\mathrm{c}$

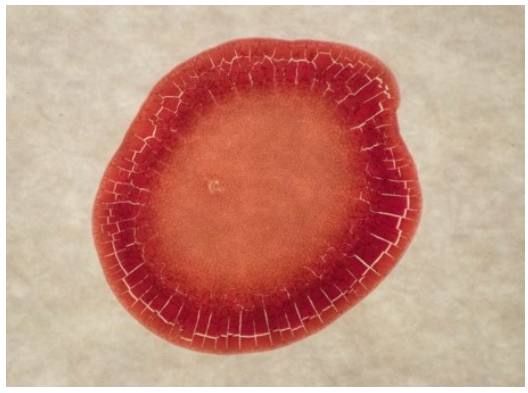

Figure 1. Comparison of two blood drops. a) Sample from a "low-normal" glucose sample. b) Converted to binary in the image analysis process. c) Sample from a "high" glucose sample. 